\title{
Research on Data-Centric Geographic Routing Algorithm for WSNs
}

\author{
Hualin Zhan ${ }^{1, a}$, Xiaochun Lai, ${ }^{2, b}$, Guoli Wang ${ }^{1, c}$, Pengfei Zuo ${ }^{1, d}$ \\ ${ }^{1}$ Communication and Electronic College, JiangXi Science \& Technology Normal University, \\ NanChang, China \\ ${ }^{2}$ Science and Technology Development Office, JiangXi Provincial Higher Education Institutions, \\ NanChang, China

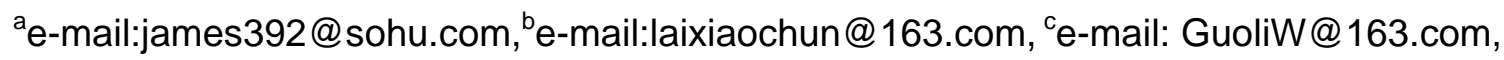 \\ de-mail:PengfeiZ@sohu.com
}

Keywords:WSNs; Data center; Geographical position; Router algorithm

\begin{abstract}
Wireless sensor networks(WSNs) can real-time collect information and monitor the objects located regional distribution network, has a broad application prospect. Research on efficient routing algorithm becomes the core problem to improve the communication connectivity, reduce transmission delay, prolong the network life cycle for WSNs. According to the application environment in this paper, the author put forward to data-centric geographic routing algorithm suitable for marine fishing nodes. Simulation results show the effectiveness of the proposed algorithm.
\end{abstract}

\section{Introduction}

WSNs is formed by lots of cheap micro sensor nodes which are deployed in the monitoring region, forming a multi hop self-organizing network by the way of wireless communication, aiming to cooperative sensing, collecting and processing network information of object in the coverage area, and sending to the observer. Routing protocol is responsible for finding the optimal path and transmitting the data packets between the source node and the destination node.

\section{Routing Protocol}

Compared with traditional network routing protocols, routing protocols in WSNs should pay more attention to energy consumption, topological information and the data center, especially to consider the application of scene. On account of thousands of different sensor networks environment and data communication mode, there are no routing mechanism suitable for all applications, hence there is necessary to design specific application mechanism to adapt to each specific application requirements. In addition, the routing mechanism of WSNs is often associated with data fusion technology, saving energy by reducing the amount of communication.

\section{Data-Centric Geographic Routing ( DCGR)}

\section{A. Application Environment}

According to the application background of this theme, there are mainly two kinds of network data transmission, including upload information from fringe node to centric node and issue a query command from the sink node to duty region, the former adopt to greedy forwarding algorithm for sending data to the next and towards the destination node, the latter take greedy forwarding algorithm toward the specific node and flood propagation algorithm in the event area. In the view of the above application scene, the author puts forward to data-centric geographical routing (DCGR) protocol. Data-centric routing is mainly responsible for passing command from the sink node to the event area, geographical routing is mainly quickly and efficiently deal with transfer the information from fringe node to centric node. 


\section{B. Principle of Routing Algorithm}

Aiming at the background of project, this paper present DCGR protocol (data center and geographical routing).Upload data from fringe node to centric node can be regarded as the spread of unicast, multi-hop,point-to-point communication by using greedy forwarding algorithm, this way has the advantages of accurate transmission, less routing hops, short delay, low energy consumption. The centric node actively send query command by using the greedy forwarding algorithm before reaching the event area and by using the flooding protocol after reaching the event area, the former can be regarded as the spread of unicast, multi-hop, point-to-point communication, the latter can be viewed as multicast, multi-hop, point-to-multipoint or multipoint-to-multipoint communication. In fact, data-centric geographical routing (DCGR) protocol is made of the greedy forwarding algorithm and the flooding protocol whether upload information or not issue information. Here separately introduce the greedy forwarding algorithm and flooding protocol.

1) Greedy Forwarding Algorithm In the Speed Characteristic

The greedy forwarding algorithm of speed characteristics in this paper and GAER algorithm of energy characteristics is different, the energy supply of the nodes is no problem because the nodes put down fishing vessel, the opposite is to seriously consider fishing vessel move speed, so the estimated price about routing algorithm should take into account normalized distance from the node to the event area and speed of nodes. Because the nodes know their positions and the geometric center locations of event area, all nodes are able to calculate distance from their own to the geometric center locations of event area. Estimate cost formula from the current node to the event area as fellow:

$$
c(N, R)=\alpha \bullet d(N, R)+(1-\alpha) v(N)
$$

Note: $c(N, R)$ shows estimate the cost from node $\mathrm{N}$ to the event region $\mathrm{R}, d(N, R)$ shows distance from node $\mathrm{N}$ to the event region $\mathrm{R}, v(N)$ shows node $\mathrm{N}$ average speed, $\alpha$ shows the scale parameter.

Transmission path from the center node create by the way of the greedy forwarding algorithm in the speed characteristics, the current node select the next-hop routing node in the neighbor region which the minimum estimate cost, and then the next-hop neighbor nodes select the next-to-next hop in the same way until the query command reach the event area. If routing cost of all nodes located the neighboring region are bigger than oneself, then it come into routing empty.

After query information reach the event area, the node in the event area do not transmit monitoring data along the reverse direction which issue query information path, do upload data by using the greedy forwarding algorithm in this paper, it mainly deal with nodes in the fishing vessel frequent topology changes, because the topology changes make reverse routing path exist no longer.

2)Flooding Routing Protocol

Flooding routing protocol is a kind of traditional network routing protocol. The flooding routing protocol does not require the maintenance of network topology and routing calculation, only required nodes broadcast forwarding packets when nodes receives the message. Flooding routing protocol should be one of the flat routing algorithm for the smaller WSNs. First, the realization of routing is a simple. Second, do not need to maintain the network topology information and do not consume extra energy for discovering the complex routing. Third, Suitable for robust applications requiring high. Flooding protocol used in this paper is consistent with most references. Figure 1 is a schematic diagram of the flooding protocol.

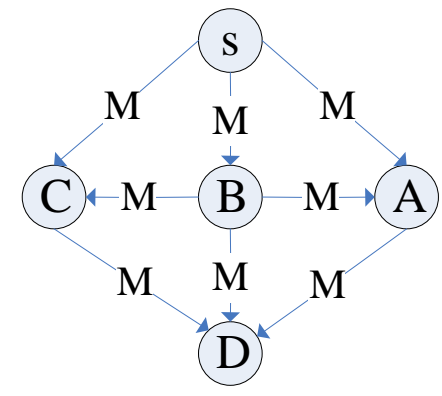

Figure 1. schematic diagram of the flooding protocol 
3)Algorithm Realization

Figure 2 give an example to illustrate principle and implementation process about DCGR algorithm.

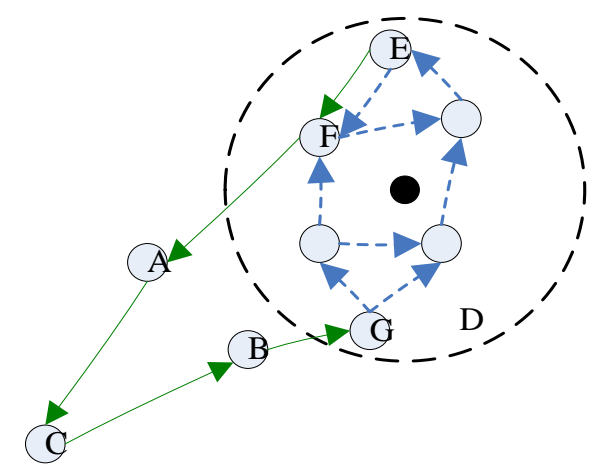

Figure 2. DCGR Algorithm Demo

Note: — shows data communication path for the speed characteristics greedy forwarding, $\rightarrow$ shows data transmission path for flooding protocol, $\mathrm{D}$ event region.

When the central node $C$ send query command to event region $\mathrm{D}$, node $\mathrm{C}$ actively RTS(request to send), node A and node B send routing reply because node A and node B meet the routing reply requirements, node $C$ receives all routing reply messages within the specified time and selects minimum estimate cost node of all reply nodes, hence ascertain node B as the next-hop node, then the query message is sent to node $\mathrm{B}$. When node $\mathrm{B}$ receives a query message, it immediately send RTS to neighbor region, node A and node $G$ send routing reply because node $A$ and node $G$ meet the routing reply requirements, but node B will select node $G$ as the next-hop node for estimate cost of node $G$ smaller than node A, then the query message is forwarded to node G. Node $G$ is confirm within the event area because distance from node $G$ to geometric center of the event area less than radius of the event area, So node $G$ directly broadcast the query message in the event area instead of RTS when node $\mathrm{G}$ receives the query message.

If node E want to send message to the centric node, node E should RTS, there will send routing reply only if node meet the routing reply requirements, then node E select node $\mathrm{F}$ as the next-hop forwarding node because node $\mathrm{F}$ has the minimum estimate cost in all routing reply nodes, next node E send message to node F, message was repeatedly send likewise until it was uploaded the central node C.

As the nodes randomly arrange and frequently mobile, the routing estimate cost continuously change. If the routing estimate cost of node is least than others in its' neighbor range, the node will fall into routing void so that the message can not be passed on. It is very important to deal with the routing void.

\section{Manage Routing Void}

If the routing estimate cost of node is least than others in its' neighbor range, the node will fall into routing void so that its' node can not receive the routing reply, thence there is appear the routing void. The essence of this situation is caused by the greedy forwarding algorithm. Although this appears less, the whole transmission can not continue as long as the routing void take place. There are put forward to two schemes to solve the routing void. The first method, if the node RTS three times, still do not receives any routing reply, illustrate that this node fall into the routing void, thence this node send request command to the centric node for adjusting network topology structure. The second method, the node RTS once more if it appears the routing void, the neighbor node also send the routing reply when it receives the special routing request even if the estimate cost of its own more than the request node, then the request node select the minimum estimate cost node in all routing reply node as the next-hop forwarding node. Comparison of two methods, reliability of the first method is not strong because the relay node likely fall into routing void again while the centric node sends a command for adjusting network topology structure, so this paper uses the second methods. 


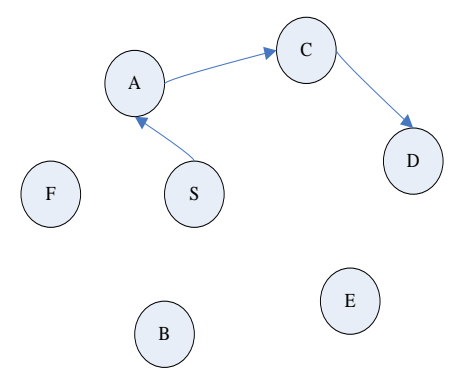

Figure 3 . the routing void

As shown in Figure 3, node $\mathrm{S}$ do not found any routing reply node in the neighbor region, the routing void will occur, at this time, node S RTS again, node A and F and B in the neighbor region send routing reply, node $S$ select the minimum estimate cost node in node $A / F / B$ as the forwarding relay node. So the problem of the routing void was preferably solved, DCGR algorithm can be further improved.

\section{DCGR Algorithm Simulation}

Figure 4 and figure 5 respectively show data transmission rate in the routing layer from node 0 to the centric node 5 in two hundred seconds by using of DCGR an GEAR. It can be found that DCGR realize the on-demand routing, the network data flow occurs in the case of sending data, thus DCGR can reduce the channel time occupied by nodes and the energy consumption, GEAR transmission rate did not fall because data are transferring in the target area by using of the flood protocol for a long period of time.

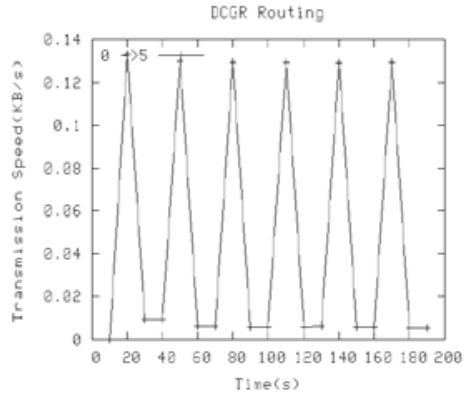

Figure 4. DCGR transmission rate

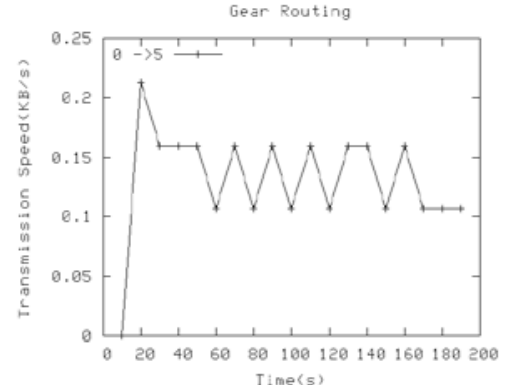

Figure 5. GEAR transmission rate

Figure 6 shows proportion of the amount about receive data and send data from node 0 to node 4 by separately using of DCGR and GEAR, that is the success rate of reach. As shown in Fig 1-6, the success rate of reach is relatively high when the number of WSNs nodes cut down, it is decreasing with the increase of WSNs nodes, but DCGR faller than GEAR. Two reasons lead to this phenomenon. First, it is very difficulty to find routing in the case of large nodes, second, the routing void cause data packet loss, while the GEAR will not drop packets as long as date is transmitted to the target area.

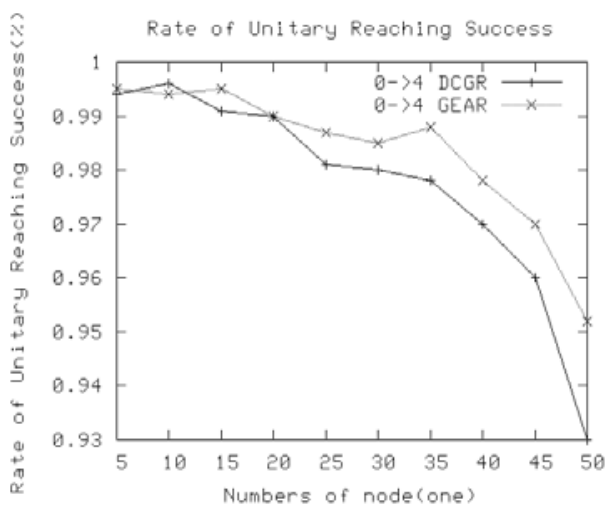

Figure 6. the normalized success rate

Figure7 respectively shows delay time from node 0 to node 4 by using of DCGR and GEAR. From that, WSNs becomes more and more complex and increase routing overhead and grow transmission delay with the increasing number of nodes, while DCGR delay time is smaller than 
GEAR in the case of same network size, and the difference of transmission delay time is more obvious with the increase of network nodes.

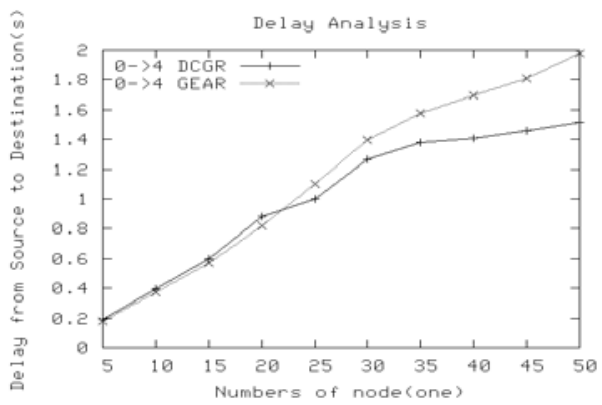

Figure7 transmission delay

\section{Conclusion}

Experiments shows that data-centric geographical routing (DCGR) is better than GEAR in many ways such as occupied channel time, energy consumption, transmission delay, transmission efficiency.

\section{References}

[1] Callaway E H. Wireless Sensor Network: Architecture and protocols [M]. CRC Press LLC, 2004: 41 62

[2] Y.Yu,D.Estrin,andR.Govindan.Geographical and Energy-Aware Routing: A Recursive Data Dissemination Protocol for Wireless Sensor Networks. UCLA Computer Science Department Technical Report, UCLA-CSD TR-01-0023, May 2001

[3] Wendi Rabiner Heinzelman, Anantha Chandrakasan, and Hari Balakrishnan.Energy-Efficient Communication Protocol for Wireless Microsensor Networks. In: HICSS Proceedings of the 33rd Hawaii International Conference on System Sciences-Volume 8-Volume 8,Washington:IEEE Computer Society,2000,vol 8 : 8020-8029

[4] S.Lindsey and C.S.Raghavendra. PEGASIS: Power Efficient GAthering in Sensor Information Systems. In:the Proceedings of the IEEE Aerospace Conference, March 2002.vol.3:1125-1130

[5] Yu Y,Govindan R, Estrin D. Geographical and energy aware routing: A recursive data dissemination protocol for wireless sensor networks. UCLA Computer Science Department Technical Report UCLA/CSD-TR-01-0023, May 2001.

[6] Shah R C, Rabaey J M. Energy aware routing for low energy ad hoc sensor networks. In: Proc IEEE Wireless Communications and Networking Conference (WCNC'02),IEEE, Volume 1,17-21 March, 2002

[7] Boukerche A, Cheng X, Linus J. Energy-aware data-centric routing in microsensor networks.In: Proc 6th ACM int'l Workshop on Modeling Analysis and Simulation of Wireless and Mobile Systems(MSWiM’03),San Diego, CA.September 19,2003.42 49.

[8] Karp B,Kung H T.GPSR: Greedy perimeter stateless routing for wireless networks.In: Proc 6th Annual Int'1 Conf on Mobile Computing and Networking(MobiCOM 2000), Boston, MA.Aug.611,2000 . 